\title{
In reply: Maspin and it's evolving role in tumor progression in systemic malignancies
}

\author{
Asru K. Sinha $\cdot$ Rajeshwary Ghosh
}

Received: 21 November 2013/ Accepted: 25 November 2013/Published online: 11 December 2013

(c) The Japanese Breast Cancer Society 2013

In reference to the letter by Dr. Kapoor [1], the author elegantly described the role of maspin synthesis in the possible contribution both in bladder and colorectal cancer. In the letter, the author claimed that the expression of maspin (gene) indicated a worst prognostic outcome in bladder and colorectal cancer.

It should be noted here that cancer is generally believed to be a disease of the gene. The heterogeneous nature of the cancer cells, particularly with different cancers, cannot be equated to uniformity in their expression of genes particularly because the expression of any gene is a multistep process. As cancer cells sometimes do undergo rapid mutation, it will almost be impossible to predict the expression of a particular gene in the pathogenesis of any cancer. For example, although the mutation of maspin genes has been claimed to result in breast cancer, the addition of nitric oxide (NO) to the in vitro reaction mixture containing $\operatorname{ER}(-)$ neutrophils from breast cancer subjects, which do not synthesize maspin in the presence of estrogen, resulted in the expression of the maspin gene supposedly that was mutated and resulted in the pathogenesis of the breast cancer [2].

Also it should be noted that the role of estrogen and progesterone in the pathogenesis of breast cancer in the context of maspin synthesis might not be extended to the cases of bladder and colorectal cancer. It should also be noted that the expression of estrogen- or progesterone-dependent synthesis of maspin was not "autonomous" in nature in that the effect of these steroids in

This reply refers to the article available at doi:10.1007/s12282-0130509-8.

A. K. Sinha $(\varangle) \cdot$ R. Ghosh

Sinha Institute of Medical Science and Technology,

288 Kendua Main Road, Baishnabghata, Garia,

Kolkata 700084, India

e-mail: asruksinha@yahoo.com the synthesis of the anti-breast cancer protein was inhibited in the presence of insulin in the system owing to "cross talk" between the insulin and the steroid receptors on the cell surface. As the systemic presence of insulin would "downregulate" the steroid receptor numbers owing to the existence of type 2 diabetes mellitus [3], the role of systemic increase or decrease of maspin synthesis in predicting the recurrence of cancers needs to be considered with appropriate caution.

However, in contrast to the negative role of maspin gene expression in colorectal cancer, as described by the letter's writer, we have found impressive good prognostic outcomes in colorectal cancer through the systemic increase of NO by using acetyl salicylic acid that increased systemic maspin synthesis [4]. Also it must be noted that the increase of NO by using acetyl salicylic acid was apparently found to reduce the metastasis of breast cancer, a notorious malignant condition with a high tendency to metastasize.

Conflict of interest The authors declare that they have no conflict of interest.

\section{References}

1. Kapoor S. Maspin and it's evolving role in tumor progression in systemic malignancies. Breast Cancer. 2013. doi:10.1007/s12282013-0509-8.

2. Ganguly Bhattacharjee K, Bhattacharyya M, Halder UC, Jana P, Sinha AK. The role of neutrophil estrogen receptor status on maspin synthesis via nitric oxide production in human breast cancer. J Breast Cancer. 2012;15(2):181-8.

3. Bhattacharjee KG, Bhattacharyya M, Halder UC, Jana P, Sinha AK. The "Cross Talk" between the receptors of insulin, estrogen and progesterone in neutrophils in the synthesis of maspin through nitric oxide in breast cancer. Int J Biomed Sci. 2012;8(2):129-39.

4. Girish GV, Sinha N, Chakraborty K, Bhattacharya G, Kahn NN, Sinha AK. Restoration by aspirin of impaired plasma maspin level in human breast cancer. Acta Oncol. 2006;45(2):184-7. 